\title{
Search for Muon Neutrino Disappearance in a Short-Baseline Accelerator Neutrino Beam
}

\author{
Yasuhiro Nakajima* \\ $\dagger$ \\ Kyoto University \\ E-mail: nakajima@scphys.kyoto-u.ac.jp
}

\begin{abstract}
Neutrino oscillations have been observed and confirmed at $\Delta m^{2} \sim 10^{-3}$ and $10^{-5} \mathrm{eV}^{2}$ with various experiments. While oscillations at other mass splittings are prohibited by the current standard model, the LSND experiment observed an excess of electron antineutrinos in a muon antineutrino beam, indicating a possible oscillation at $\Delta m^{2} \sim 1 \mathrm{eV}^{2}$. To test the oscillation at $\Delta m^{2} \sim 1 \mathrm{eV}^{2}$, we search for muon neutrino disappearance using the Fermilab Booster Neutrino beamline and two experiments, SciBooNE and MiniBooNE. The neutrino fluxes are measured in the SciBooNE and MiniBooNE detectors, located at $100 \mathrm{~m}$ and $540 \mathrm{~m}$ downstream from the neutrino production target, respectively. We collected beam data from June 2007 through August 2008 with SciBooNE, and over a five year period with MiniBooNE. A preliminary sensitivity for a joint $v_{\mu}$ disappearance search is presented.
\end{abstract}

35th International Conference of High Energy Physics

July 22-28, 2010

Paris, France

\footnotetext{
* Speaker.

$\dagger$ The author was supported by Japan Society for the Promotion of Science, and by the Grant-in-Aid for the Global COE Program "The Next Generation of Physics, Spun from Universality and Emergence” from the MEXT of Japan.
} 
An observation of a data excess of $\bar{v}_{e}$ in a $\bar{v}_{\mu}$ beam by the LSND experiment [1] initiated interests of a possible neutrino oscillation at $\Delta m^{2} \sim 1 \mathrm{eV}^{2}$. In this paper, we discuss a search for $v_{\mu}$ disappearance at $\Delta m^{2} \sim 1 \mathrm{eV}^{2}$ using data from both the SciBooNE [2] and the MiniBooNE [3] experiments, where the SciBooNE detector is used to constrain flux and cross-section uncertainties.

The experiments use the Booster Neutrino Beam (BNB) at Fermilab [4]. An intense muon neutrino beam with mean energy of $\sim 0.8 \mathrm{GeV}$ are produced and detected at the SciBooNE and MiniBooNE detectors. The SciBooNE detector is located $100 \mathrm{~m}$ downstream from the neutrino production target. The main component of the detector complex is a fully active fine grained scintillator tracker, SciBar. The detector itself is the neutrino target $(\mathrm{CH})$ and its fiducial volume is 10.6 tons. The MiniBooNE detector is located $440 \mathrm{~m}$ downstream from the SciBooNE detector, and is a $12 \mathrm{~m}$ diameter spherical tank filled with 800 tons of mineral oil $\left(\mathrm{CH}_{2}\right)$.

First, we measure energy dependent $v_{\mu}$ inclusive charged current (CC) interaction rates (product of flux and cross section) by fitting muon kinematics using SciBooNE data [2]. Then, we use the result of the CC interaction measurement to tune the $v_{\mu}$ interaction rate at MiniBooNE. Systematic uncertainties are carefully estimated by evaluating the correlation between SciBooNE and MiniBooNE data. Finally, we test the oscillation hypothesis assuming the mixing between 2 neutrino flavors; $v_{\mu}$ and $v_{x}$. Figure 1 shows the $90 \% \mathrm{CL}$. sensitivity for the $v_{\mu}$ disappearance. We find that the expected sensitivity directly supersedes the MiniBooNE only $v_{\mu}$ disappearance result [3], as substantial flux and cross section uncertainties have been reduced. The results of this analysis will be released soon.

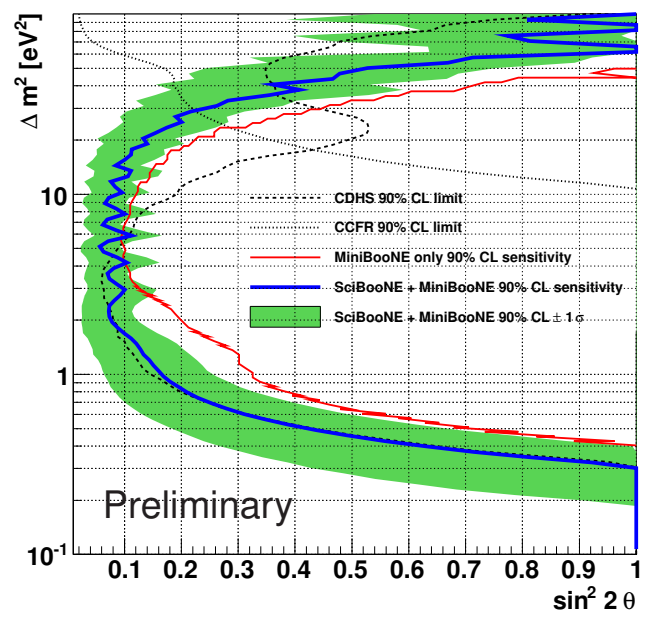

Figure 1: The expected sensitivity for $v_{\mu}$ disappearance. The dotted curve shows the $90 \%$ CL limits from CDHS [5] and CCFR [6] experiments. The thin solid curve is the MiniBooNE-only $90 \% \mathrm{CL}$ sensitivity. The thick solid curve and the filled region are the $90 \%$ CL sensitivity and $\pm 1 \sigma$ band from SciBooNE-MiniBooNE joint analysis, respectively.

\section{References}

[1] A. Aguilar et al., Phys. Rev. D 64 (2001) 112007.

[2] Y. Nakajima et al. (SciBooNE Collaboration), arXiv:1011.2131.

[3] A. A. Aguilar-Arevalo et al. (MiniBooNE Collaboration), Phys. Rev. Lett. 103 (2009) 061802.

[4] A. A. Aguilar-Arevalo et al. (MiniBooNE Collaboration), Phys. Rev. D 79 (2009) 072002.

[5] F. Dydak et al., Phys. Lett. B134 (1984) 281.

[6] I. E. Stockdale et al., Phys. Rev. Lett. 52 (1984) 1384. 